\title{
Effect of nerve mobilization with intermittent cervical segment traction on pain, range of motion, endurance, and disability of cervical radiculopathy
}

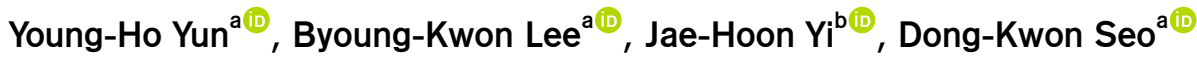 \\ ${ }^{a}$ Department of Physical Therapy, College of Medical Science, Konyang University, Daejeon, Republic of Korea \\ ${ }^{\mathrm{b}}$ Department of Smart Rehabilitation Care, Pyeongtaek University, Pyeongtaek, Republic of Korea
}

Objective: This study aimed to evaluate the effects of the Kaltenborn-Evjenth concept of nerve mobilization combined with intermittent cervical segment traction (ICST) on pain, Neck Disability Index (NDI) scores, range of motion (ROM) and endurance in persons with cervical radiculopathy (CR).

Design: Two-group pretest-posttest design.

Methods: Thirty subjects participated in this study and were randomly assigned to two groups. The ICST group (n=15) was performed simultaneously with nerve mobilization and cervical traction for the segment with cervical pain at the same time. The intermittent cervical total traction (ICTT) group $(n=15)$ performed nerve mobilization and cervical traction for the whole cervical area at the same time. In this study, outcome measures such as the Visual Analog Scale (VAS), NDI, ROM, endurance (cranio-cervical flexion test), and passive intervertebral motion performed before and 4 weeks after the experiment were compared to investigate the effects of each intervention.

Results: In both groups, there were significant differences in the VAS, NDI scores, and endurance, and there were significant differences between the two groups except for endurance $(p<0.05)$. In the ICST group, significant differences were found in all ROM, and in the ICTT group, significant differences were found in only extension, and there were significant differences between the two groups $(p<0.05)$.

Conclusions: The ICST group showed more improvement than the ICTT group in pain, NDI scores and ROM. Moreover, our findings show that the ICST could be used as a new strategy for manual therapy in persons with CR.

Key Words: Cervical radiculopathy, Neural mobilization, Traction

\section{Introduction}

In the modern age of fast information, many people develop musculoskeletal system diseases that cause cervical problems due to prolonged computer use, assumption of inappropriate working postures, and the performance of repetitive tasks [1]. Cervical radiculopathy (CR), the most common problem of the cervical spine, is a degenerative disease of the cervical spine caused by a space-occupying lesion resulting from pathological problems in the cervical nerve root, and it reportedly has 83.2 cases per 100,000 people cases per year [2].

Common causes include herniation of the intervertebral disc, spondylosis, cervical spine instability, trauma, osteophytosis, and, rarely, oncological problems, resulting in narrowing of space in the vertebral foramen where the cervical nerve root emerges or direct compression of the cervical nerve root where mechanical and chemical irritation is applied around the root to leading to inflammation of the nerve root, and other causes such as neuritis, edema, hypoxia, is-

Received: 2 September, 2020 Revised: 24 September, 2020 Accepted: 25 September, 2020

Corresponding author: Dong-Kwon Seo (ORCID https://orcid.org/0000-0002-3328-4922)

Department of Physical Therapy, College of Medical Science, Konyang University, 158 Gwanjeodong-ro, Daejeon 35365, Republic of Korea Tel: 82-42-600-8457 Fax: 82-42-600-8408 E-mail: dkseo77@konyang.ac.kr

(c) This is an Open-Access article distributed under the terms of the Creative Commons Attribution Non-Commercial License (http://creativecommons.org/licenses/ by-nc/4.0) which permits unrestricted non-commercial use, distribution, and reproduction in any medium, provided the original work is properly cited.

Copyright $@ 2020$ Korean Academy of Physical Therapy Rehabilitation Science 
chemia, fibrosis, and decrease in nerve mobility [3].

Manual therapy applied to patients with CR includes cervical traction. Cervical traction increases the vertebral body gap, increases the vertebral foramen, reduces nerve root compression, increases blood circulation, and relaxes the surrounding muscles to restore vertebral mobility. Cervical traction is reportedly effective in that it alleviates pain, and in a previous study, the group of patients with CR who received manual traction or mechanical traction showed improved pain and functionality compared to the group with only general rehabilitation [4].

Nerve mobilization is presented as an assessment of CR and a therapeutic intervention method. This increases the flexibility of the nerve to decrease the dynamic sensitivity of the nervous system and increases blood flow to indicate pain relief. Moreover, the increased joint mobility of the pain increases the dynamic range of motion (ROM) and increases the dynamic adaptability of the nervous system without resistance. It has been reported to help move the body $[5,6]$.

In previous studies, opinions have been divided on cervical traction with nerve mobilization applied to patients with CR. The effectiveness of simultaneously applying these techniques in people with CR has been investigated previously, but the study methods comparing cervical segment traction and cervical total traction are inadequate [7].

This study aimed to investigate the effects of the Kaltenborn-Evjenth (K-E) concept of nerve mobilization combined with intermittent cervical segment traction (ICST) on pain, joint ROM, neck disability index (NDI) scores, and craniocervical flexion test (CCFT) in patients with CR. When nerve root compression and inflammation occur by applying mechanical and chemical irritation to the vertebral foramen where the cervical nerve root exits, it causes neuritis, edema, fibrosis, and reduces nerve mobility [5]. Therefore, this study intended to identify the effects of ICST on pain, NDI scores, ROM, CCFT, and passive intervertebral motion, in order to provide patients with $\mathrm{CR}$ with the appropriate treatment methods, as well as to investigate for the difference between the K-E ICST group and the intermittent cervical total traction with nerve mobilization (ICTT) group.

\section{Methods}

\section{Participants}

Thirty-five patients who were treated for CR at the Madu Orthopedic hospital in Gyeonggi-do were recruited for this study. Of these 35 patients, 30 had satisfied with the selection conditions, understood the purpose of the study, and voluntarily signed the consent form.

Prior to the experiment, all subjects received the full explanation of the experiment and provided voluntary consent. This study was approved by the Institutional Review Board of Konyang University (IRB No. KYU-2020-046-01) based on the Helsinki Declaration.

\section{Intervention}

The treatment for those in the ICST group was performed in supine position, which did not cause the subjects to experience any neck pain. In addition, the neck position was set so that there was 0 degrees of flexion, lateral flexion, and rotation. The therapist stood on the cranial side, placing the radial side of both index fingers from the posterior arch of the vertebra to the left and right surfaces of the spinous process, and the index finger formed an angle of 45 degrees from the treatment table. The therapist's index finger held the posterior arch of the vertebra, below which the traction occurred. By moving the pelvis backward, the tension of the belt was transmitted to the vertebra. The belt around the hand helped to hold the vertebral arch firmly and pull in the direction perpendicular to the treatment plane. When the starting position was achieved, ICST was performed, and nerve mobilization of the upper extremity was conducted by the patients themselves. Cervical spine traction was classified into three grades, and cervical spine traction was applied using grade II. The ICST was maintained for 1 minute, starting from the glenohumeral joint external rotation, 90-degrees of abduction, elbow flexion, wrist and finger extension, and elbow extension, wrist and finger extension. The treatment provider performed painless cervical traction and started cervical traction at grade II. Regarding the application of nerve mobilization, the glenohumeral joint was ini-

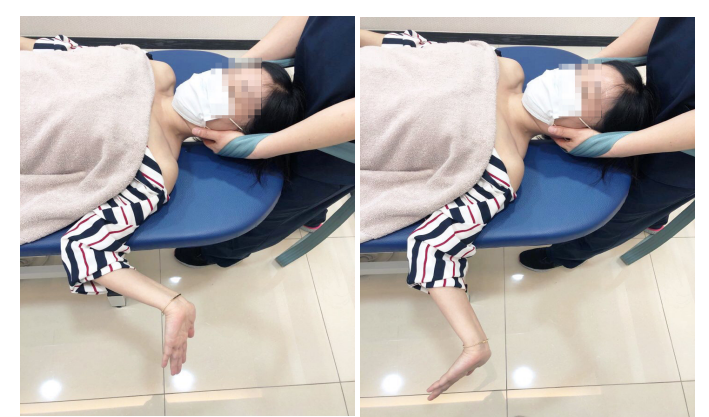

Figure 1. Intermittent cervical segment traction with nerve mobilization. 
tially placed in the neutral position and slowly moved to 90-degrees of abduction and maximal external rotation, depending on the degree of apparent neuromechanical sensitivity. The glenohumeral joint was initially at 0 degrees of abduction and external rotation for participants with high neuromechanical sensitivity, whereas the glenohumeral joint was positioned at 90-degrees of abduction and maximal external rotation for participants with low neuromechanical sensitivity. The treatment in the ICTT group was performed in entire cervical spine as a whole, and the other process is the same as ICST group.

\section{Assessment tools and experimental methods}

The study variables were the participants' pain index (assessed using the visual analog scale, VAS), NDI, ROM, and deep flexor muscle CCFT was tested before the treatment. Using the Internet program (Research randomizer; Geoffrey C. Urbaniak and Scott Plous, Lancaster, PA, USA), two random groups were formed, and 15 people in the ICST group were randomly assigned to experimental group 1, while 15 people in the ICTT group were randomly assigned to experimental group 2. The arbitration time of each group was conducted three times a week for a total of 50 minutes/day, and the ICST and ICTT groups were composed of seven sets (1-minute treatment, 1-minute rest) and 35 minutes of conservative physical therapy were applied. All subjects underwent conservative physical therapy three times a week for 4 weeks, which included superficial heat treatment for 20 minutes followed by transcutaneous electrical nerve stimulation. The frequency was set at $60 \mathrm{HZ}$ applied for 15 minutes. After treatment, the subjects were divided into the ICST and ICTT groups. Afterwards, VAS, neck disability, joint ROM and deep flexor muscle endurance tests were assessed after 4 weeks. Each group was treated for 4 weeks by a physical therapist with a musculoskeletal K-E orthopaedic manual therapy qualification and more than 10 years of treatment and evaluation experience before the experiment. Each examination was conducted by a physical therapist with more than 7 years of experience in musculoskeletal treatment and evaluation who did not participate in the experiment.

Pain was assessed using a numerical pain scale, which is a 'pain graph' with numbers from 0 to 10 , with zero representing no pain at all, and 10 representing the highest possible pain intensity [8]. The test-retest reliability is $0.57-0.83$ [9]. The Korean-language version of the NDI (K-NDI) was used to measure the neck-pain intensity and disabilty [10]. It was created based on the Oswestry index's 10-question questionnaire, which evaluates daily life limitations related to back pain, pain intensity, daily life, lifting, reading, headache, concentration, work, driving, sleep, and leisure, selected from one of six items for each of the 10 items (0-5). The NDI score is the sum total of the items, and the higher the score, the greater the disability associated with cervical abnormalities. The internal concordance is 0.82 , and inter-rater confidence is 0.93 [10]. To measure the ROM of the neck, a joint ROM instrument was used, and the parameters were measured in degrees of ervical flexion and extension, left and right lateral flexion, and left and right rotation. Subjects were comfortably seated in a chair and started at the front for measurement. The intra-measurement reliability was $0.89-0.98$ and the inter-measurement reliability was $0.76-0.98$ [11]. The deep neck flexor test was used to selectively test the deep muscles of the neck and the muscular dndurance of the muscle group. The time to maintain $60 \%$ of the maximal voluntary contractile force (MVC) of the deep neck flexors was measured, and a pressure biofeedback device (Chattanooga stabilizer pressure biofeedback; Chattanooga Group Inc., Chattanooga, TN, USA) was used to measure the MVC of the neck flexors. After all measurements were performed three times, the average value of each measurement was used in this study, and a 2-minute break was provided between each execution, and the measurement unit was mmHg. The intra-measurer reliability was $0.82-0.89$, and the inter-measurer reliability was $0.71-0.75$ [12].

\section{Data analysis}

For statistical analysis in this study, IBM SPSS Statistics for Windows, Version 22.0 (IBM Co., Armonk, NY, USA) was used. The Shapiro-Wilk test was used to test the normality of every group variable, and the normal distribution was confirmed. The Chi-squared test and independent t-test were used to compare the general characteristics of the subjects, and there was no significant difference as a result of using the independent sample t-test to test the homogeneity of the pre-dependent variables of each group. The data appeared to be homogeneous. Paired t-tests were used to compare differences before and after training within groups, while independent sample t-tests were used to compare the differences between groups. All statistical significance levels were set to $p<0.05$. 


\section{Results}

The general characteristics are shown in Table 1 . Participant characteristics across groups were relatively similar. In both groups, the paired t-test revealed significant differences in VAS, NDI, and endurance and there were significant differences between the two groups except for endurance $(p<0.05$; Table 2$)$. In ICST group, the paired t-test revealed significant differences in all ROM, and in ICTT group, the paired t-test revealed significant differences only in extension, and there were significant differences between the two groups $(p<0.05$; Table 3$)$.

\section{Discussion}

In this study, cervical traction was applied to increase the spacing of the vertebral body that can directly apply mechanical pressure to the nerve when the nerve is moving in patients with chronic $\mathrm{CR}$, while simultaneously improving the nutrient supply and normal length and mobility of the nervous system. The effect of applying a slider technique that does not cause stress due to stretching of the nerve during nerve mobilization has been confirmed [13].

In the study by Savva and Giakas [14], pain was decreased by simultaneously applying cervical manual traction and nerve mobilization to patients with $\mathrm{CR}$, reducing compression of the cervical nerve root, and restoring nervous system mobility, resulting in CR pain. It was able to confirm

Table 1. General characteristics of the subjects

\begin{tabular}{lrrr}
\hline Variable & ICST $(\mathrm{n}=15)$ & ICTT $(\mathrm{n}=15)$ & $\chi^{2} / \mathrm{t}(p)$ \\
\hline Sex & & & $0.682(0.682)$ \\
Male & $3(20.0)$ & $5(33.3)$ & $0.214(0.832)$ \\
Female & $12(80.0)$ & $10(66.7)$ & $-1.302(0.203)$ \\
Age $(\mathrm{y})$ & $47.07(2.99)$ & $46.80(3.80)$ & $0.385(0.703)$ \\
Height & $161.53(7.30)$ & $165.60(9.64)$ & $0.248(0.884)$ \\
Weight & $61.73(14.04)$ & $59.93(11.44)$ & \\
Level & & $2(13.3)$ & \\
C4 & $3(20.0)$ & $11(73.4)$ & \\
C5 & $10(66.7)$ & $2(13.3)$ & \\
C6 & $2(13.3)$ &
\end{tabular}

Values are presented as $\mathrm{n}(\%)$ or mean (SD).

ICST: intermittent cervical segment traction, ICTT: intermittent cervical total traction.

Table 2. Comparison of pain, NDI, and endurance between two groups

\begin{tabular}{lcrr}
\hline \multicolumn{1}{c}{ Variable } & ICST $(\mathrm{n}=15)$ & $\mathrm{ICTT}(\mathrm{n}=15)$ & $\mathrm{t}(p)$ \\
\hline VAS (score) & & & $0.503(0.619)$ \\
Pre-test & $7.00(1.36)$ & $6.73(1.53)$ & $-3.674(0.001)$ \\
Post-test & $4.00(1.36)^{*}$ & $5.80(1.32)^{*}$ & $-3.661(0.001)$ \\
Change value & $-3.00(1.77)$ & $-0.93(1.28)$ & $0.378(0.709)$ \\
NDI (score) & & & $-3.374(0.002)$ \\
Pre-test & $28.20(4.38)$ & $27.60(4.32)$ & $-3.989(<0.001)$ \\
Post-test & $17.93(4.33)^{*}$ & $24.00(5.45)^{*}$ & $-0.603(0.552)$ \\
Change value & $-10.27(5.39)$ & & $1.886(0.070)$ \\
Endurance (score) & & & $2.38(0.72)$ \\
Pre-test & $8.33(0.82)$ & $9.09(1.27)$ & $0.71(0.89)$ \\
Post-test & $9.20(1.17)$ & & $0.019)$ \\
Change value & $0.86(0.82)$ & &
\end{tabular}

Values are presented as mean (SD).

ICST: intermittent cervical segment traction, ICTT: intermittent cervical total traction, VAS: visual analog scale, NDI: neck disability index. *Statistical difference within group $(p<0.05)$. 
Table 3. Comparison of range of motion between two groups

$(\mathrm{N}=30)$

\begin{tabular}{|c|c|c|c|}
\hline Range of motion & $\operatorname{ICST}(n=15)$ & $\operatorname{ICTT}(\mathrm{n}=15)$ & $\mathrm{t}(p)$ \\
\hline \multicolumn{4}{|l|}{ Flex (score) } \\
\hline Pre-test & 43.47 (4.29) & $44.38(6.35)$ & $-0.603(0.552)$ \\
\hline Post-test & $50.22(3.67)^{*}$ & $47.65(3.81)^{*}$ & $1.886(0.070)$ \\
\hline Change value & $6.75(4.33)$ & $3.27(3.95)$ & $2.493(0.019)$ \\
\hline \multicolumn{4}{|l|}{ EXT (score) } \\
\hline Pre-test & $56.01(3.21)$ & $54.58(3.13)$ & $1.239(0.226)$ \\
\hline Post-test & $65.80(3.34)^{*}$ & $60.43(5.89)^{*}$ & $3.070(0.005)$ \\
\hline Change value & $9.79(3.22)$ & $5.85(4.80)$ & $2.637(0.013)$ \\
\hline \multicolumn{4}{|l|}{ Rt. SB (score) } \\
\hline Pre-test & $36.55(2.68)$ & $35.29(2.66)$ & $1.291(0.226)$ \\
\hline Post-test & $40.47(2.88)^{*}$ & $36.41(2.35)^{*}$ & $4.336(<0.001)$ \\
\hline Change value & $4.02(3.10)$ & $1.12(1.29)$ & $3.347(0.003)$ \\
\hline \multicolumn{4}{|l|}{ Lt. SB (score) } \\
\hline Pre-test & $31.62(2.53)$ & $31.43(3.33)$ & $0.0185(0.854)$ \\
\hline Post-test & $38.12(1.74)^{*}$ & $33.28(2.71)^{*}$ & $5.826(<0.001)$ \\
\hline Change value & $6.49(3.11)$ & $1.85(2.71)$ & $4.361(<0.001)$ \\
\hline \multicolumn{4}{|l|}{ Rt. Rot (score) } \\
\hline Pre-test & $57.81(3.93)$ & $59.91(5.40)$ & $-1.222(0.232)$ \\
\hline Post-test & $64.15(3.38)^{*}$ & $61.85(4.67)^{*}$ & $1.544(0.134)$ \\
\hline Change value & $6.38(3.72)$ & $1.94(2.95)$ & $3.595(0.001)$ \\
\hline \multicolumn{4}{|l|}{ Lt. Rot (score) } \\
\hline Pre-test & $61.45(1.73)$ & $62.08(1.55)$ & $-1.045(0.305)$ \\
\hline Post-test & $66.67(2.36)^{*}$ & $63.26(1.51)^{*}$ & $4.700(<0.001)$ \\
\hline Change value & $5.21(3.12)$ & $1.18(1.60)$ & $4.454(<0.001)$ \\
\hline
\end{tabular}

Values are presented as mean (SD).

ICST: intermittent cervical segment traction, ICTT: intermittent cervical total traction, Flex: flexion, EXT: extention, Rt. SB: right side bending, Lt. SB:left side bending, Rt. Rot: right rotation, Lt. Rot: left rotation.

* Statistical difference within group $(p<0.05)$.

the analgesic effect obtained by preventing factors that could exacerbate and stimulate the mechanoreceptors. In this study, subjects in the cervical segment traction with nerve mobilization group showed greater reduction of pain than the subjects in the total traction with nerve mobilization group $(p<0.05)$.

In a previous study, Cleland, et al. [15] showed an improvement in the function disability index of patients with an intervention method combining cervical traction and muscle strengthening exercises for CR. This is a study of a complex treatment method along with cervical traction. It was confirmed that the disability index was significantly reduced in the group with nerve mobilization with cervical traction. In this study, there was a greater significant decrease in NDI scores in the ICST group than the ICTT group $(p<0.05)$.

Several previous studies reported that cervical joint ROM was significantly decreased in people with cervical spine pain, and manual therapy was mainly used to increase joint
ROM and decrease pain [16].

In this study, it was confirmed that the ICST group that included the combination of the two treatment methods showed a significant increase in cervical flexion and extension ROM, left and right side-bending, and left and right rotation than the ICTT group $(p<0.05)$. Nee and Butler [17] suggested that nerve mobilization stimulates mechanoreceptors to increase nerve sliding and nerve root distance, thereby improving nerve mobility.

Compared to healthy individuals, in patients with neck pain, cervical spine control capabilities decrease due to weakness of the longus colli and longus capitis, which are the deep muscles. Moreover, activity is limited due to pain and exercise capacity decreases, resulting in loss of deep muscle control [18]. In this study, both groups showed an increase in deep flexor endurance, but there was no significant difference when comparing the changes between the two groups $(p<0.05)$.

This seems to be the result of recovery of the deep muscle 
power control ability and cervical spine movement that was previously lost or limited due to pain and decrease in the amount of exercise rather than due to the increase in actual muscle power increase.

In this study, the joints with dysfunction of more than one segment in cervical motion were selected based on this theoretical background.

It has been reported that general traction can increase stress placed onto the posterior fiber of the ring fiber and cause fiber rupture if the traction load exceeds the optimal amount. Conversely, segmental traction decreases the stress placed onto the posterior fiber of the ring fiber and decreases the pressure placed on the disc. Therefore, it is recommended to carefully apply general traction to patients with intervertebral pathology [19].

As the results of this study suggested, the ICST group showed more improvement than the ICTT group in pain, NDI scores and ROM. Moreover, our findings show that ICST could be used as a new strategy for manual therapy in patients with $\mathrm{CR}$.

In this study, it was found that ICST was effective for pain, neck disability, ROM and CCFT in patients with CR. However, due to the short duration of the experiment, there were limitations in generalizing the results of the study. Moreover, the patient's age range was narrow and the number of participants was small. In addition, objective measures were not made to determine whether it is applicable to patients with acute and bilateral CR, muscle weakness, dysesthesia, and radiation pain of the upper extremity. Objective measurement of the instability segment of the cervical spine was not possible, and muscle power for the deep flexors was not measured. In subsequent studies, continuous research should be done by supplementing the above limitations.

\section{Conflict of Interest}

The authors declared no potential conflicts of interest with respect to the authorship and/or publication of this article.

\section{References}

1. Larsson B, Søgaard K, Rosendal L. Work related neck-shoulder pain: a review on magnitude, risk factors, biochemical characteristics, clinical picture and preventive interventions. Best Pract Res Clin Rheumatol 2007;21:447-63.

2. Eubanks JD. Cervical radiculopathy: nonoperative management of neck pain and radicular symptoms. Am Fam Physician 2010; $81: 33-40$

3. Thoomes EJ. Effectiveness of manual therapy for cervical radiculopathy, a review. Chiropr Man Therap 2016;24:45.

4. Jellad A, Ben Salah Z, Boudokhane S, Migaou H, Bahri I, Rejeb $\mathrm{N}$. The value of intermittent cervical traction in recent cervical radiculopathy. Ann Phys Rehabil Med 2009;52:638-52.

5. Shacklock M. Neural mobilization: a systematic review of randomized controlled trials with an analysis of therapeutic efficacy. J Man Manip Ther 2008;16:23-4.

6. Neto T, Freitas SR, Marques M, Gomes L, Andrade R, Oliveira R. Effects of lower body quadrant neural mobilization in healthy and low back pain populations: a systematic review and meta-analysis. Musculoskelet Sci Pract 2017;27:14-22.

7. Hassan F, Osama M, Ghafoor A, Yaqoob MF. Effects of oscillatory mobilization as compared to sustained stretch mobilization in the management of cervical radiculopathy: a randomized controlled trial. J Back Musculoskelet Rehabil 2020;33: 153-8.

8. McCaffery M, Pasero C. Teaching patients to use a numerical pain-rating scale. Am J Nurs 1999;99:22.

9. Herr KA, Spratt K, Mobily PR, Richardson G. Pain intensity assessment in older adults: use of experimental pain to compare psychometric properties and usability of selected pain scales with younger adults. Clin J Pain 2004;20:207-19.

10. Song KJ, Choi BW, Choi BR, Seo GB. Cross-cultural adaptation and validation of the Korean version of the neck disability index. Spine 2010;35:E1045-9.

11. Audette I, Dumas JP, Côté JN, De Serres SJ. Validity and between-day reliability of the cervical range of motion (CROM) device. J Orthop Sports Phys Ther 2010;40:318-23.

12. Falla DL, Campbell CD, Fagan AE, Thompson DC, Jull GA. Relationship between cranio-cervical flexion range of motion and pressure change during the cranio-cervical flexion test. Man Ther 2003;8:92-6.

13. Wainner RS, Fritz JM, Irrgang JJ, Boninger ML, Delitto A, Allison S. Reliability and diagnostic accuracy of the clinical examination and patient self-report measures for cervical radiculopathy. Spine 2003;28:52-62.

14. Savva C, Giakas G. The effect of cervical traction combined with neural mobilization on pain and disability in cervical radiculopathy. A case report. Man Ther 2013;18:443-6.

15. Cleland JA, Whitman JM, Fritz JM, Palmer JA. Manual physical therapy, cervical traction, and strengthening exercises in patients with cervical radiculopathy: a case series. J Orthop Sports Phys Ther 2005;35:802-11.

16. Boyles R, Toy P, Mellon J Jr, Hayes M, Hammer B. Effectiveness of manual physical therapy in the treatment of cervical radiculopathy: a systematic review. J Man Manip Ther 2011;19:135-42.

17. Nee RJ, Butler D. Management of peripheral neuropathic pain: integrating neurobiology, neurodynamics, and clinical evidence. Phys Ther Sport 2006;7:36-49.

18. Jull GA, O'Leary SP, Falla DL. Clinical assessment of the deep cervical flexor muscles: the craniocervical flexion test. $\mathrm{J}$ Manipulative Physiol Ther 2008;31:525-33.

19. Park WM, Kim K, Kim YH. Biomechanical analysis of two-step traction therapy in the lumbar spine. Man Ther 2014;19:527-33. 